\title{
A Utilização dos Videogames Ativos no Tratamento não Farmacológico da Diabetes Mellitus em Idosos: Uma Revisão Integrativa
} The Use of Active Videogames in the Non-Pharmacological Treatment of Diabetes
Mellitus in the Elderly: An Integrative Review

\author{
RAPHAEL JOSÉ PERRIER-MELO' \\ JORGE LUIZ DE BRITO-GOMES',2 \\ MANOEL DA CUNHA COSTA ${ }^{1,3}$
}

\section{RESUMO}

Objetivo: Analisar a utilização dos videogames ativos (VGAs) no tratamento não medicamentoso de idosos com Diabetes Mellitus tipo 2 por meio de uma revisão integrativa. Material e Métodos: Trata-se de uma revisão integrativa, a qual foi realizada uma pesquisa nas bases de dados: Bireme, PubMed, Science Direct e Web of Knowledge, com os descritores e termos: "exergames", "exercise games", "video game", "virtual reality", "diabetes mellitus" e "glycosyleted hemoglobin". Resultados: Após a análise inicial de leitura do título e resumo dos artigos, foram selecionados 415 estudos. Em seguida, foram excluídos 411 manuscritos por não se adequarem aos critérios de seleção da pesquisa. Posteriormente, foram considerados elegíveis para a segunda fase desta revisão quatro estudos, no qual, dois foram excluídos por serem duplicatas. Pode-se verificar que entre 10 e 12 semanas de intervenção com VGAs é possível proporcionar melhoras sobre a aptidão física e valores de hemoglobina glicada em idosos diagnosticados com Diabetes Mellitus tipo 2. Conclusão: Verificou-se um pequeno número de publicações realizadas com videogames ativos no tratamento da Diabetes Mellitus tipo 2. No entanto, os videogames ativos apresentam características positivas no tratamento não farmacológico do Diabetes Mellitus tipo 2, pois possibilitam diminuição nos valores da hemoglobina glicada e melhoras significativas no condicionamento físico após um período de no mínimo dez semanas de intervenção.

\section{DESCRITORES}

Jogos de Vídeo. Diabetes Mellitus. Glicemia.

\begin{abstract}
Objective: To analyze the use of active videogames (AVGs) in the non-pharmacological treatment of elderly with type 2 diabetes mellitus. Material and Methods: This was an integrative review based on searches in the following databases: Bireme, PubMed, Science Direct and Web of Knowledge, using the descriptors and terms: "exergames", "exercise games", "video game", "virtual reality", "diabetes mellitus" and "glycosylated hemoglobin". Results: A total of 415 articles were selected after initial reading of title and abstract. Of these, 411 articles were excluded for not meeting the inclusion criteria. Four studies were considered eligible for the second phase of this review, of which two were excluded because they were duplicates. We observed that intervention with AVGs for 10-12 weeks provides improvements in physical fitness and glycosylated hemoglobin levels in elderly diagnosed with type 2 diabetes. Conclusion: There was a small number of publications addressing the use of active videogames for the treatment of type 2 diabetes mellitus. However, active videogames have shown positive effects in the non-pharmacological treatment of such disease as they contribute to decreased levels of glycosylated hemoglobin as well as to significant improvements in physical fitness after a 10-week minimum intervention period.
\end{abstract}

\section{DESCRIPTORS}

Video games. Diabetes Mellitus. Blood Glucose.

1 Membro do Laboratório de Avaliação da Performance Humana [La]pH da Escola Superior de Educação Física da Universidade de Pernambuco ESEF/UPE, Recife, PE, Brasil.

2 Professor da Universidade Federal de Pernambuco - CAV/ Vitória de Santo Antão-PE, Brasil.

3 Docente do Programa de Pós-Graduação em Educação Física - Universidade de Pernambuco/Universidade Federal da Paraíba. Professor Adjunto da Escola Superior de Educação Física da Universidade de Pernambuco - ESEF/UPE, Recife, PE, Brasil. 
$\mathrm{O}$ processo de envelhecimento é um acontecimento existente na vida de todo ser humano, marcado por provocar alterações nas funções psicológicas, sociais e fisiológicas ${ }^{1}$. Mudanças que comprometem a participação dessa população em atividades da vida diária, favorecendo a redução da massa muscular, a qual apresenta influência significativa na diminuição do nível de aptidão física, aumentando o risco de quedas, fraturas e do desenvolvimento e/ou agravamento das doenças crônicas degenerativas não transmissíveis, como é o caso da Diabetes Mellitus $(\mathrm{DM})^{2}$.

A DM é caracterizada por uma síndrome de etiologia múltipla, causada pela insuficiência e ou ausência do hormônio insulina, como consequência gera um desequilíbrio glicêmico ${ }^{3,4}$. A DM pode ser classificada como diabetes mellitus tipo 1 (DM 1) (insulinodependente), quando há deficiência na produção de insulina, e diabetes mellitus tipo 2 (DM 2) (não insulinodependente), caracterizado por ter resistência à insulina e altos níveis glicêmicos ${ }^{5}$.

Fatores de risco modificáveis como inatividade física, hábitos alimentares inadequados, consumo de tabaco e o etilismo, contribuem para o desenvolvimento da DM tipo $2^{6}$, e na maior participação em atividades sedentárias de lazer, tais como: assistir TV, jogar videogame e utilizar computador. Assim, nota-se que tais comportamentos quando não controlados causam prejuízos nos níveis glicêmicos, podendo proporcionar diminuição da capacidade funcional e da expectativa de vida $^{7}$.

Dessa forma, vários tipos de intervenções são testadas no combate e tratamento à DM, com o objetivo de manter níveis adequados sobre o comportamento da glicemia. Pois, observa-se que durante a realização de exercícios aeróbios ${ }^{8}$ e resistidos ${ }^{9}$, ocorre a captação de glicose pelas células musculares de forma aguda, ${ }^{10,11}$ tal mecanismo favorece na diminuição da glicose circulante na corrente sanguínea. Dessa forma, ao realizar tais exercícios de maneira contínua a longo prazo, haverá um aumento na sensibilidade à insulina, consequentemente maior captação e utilização deste carboidrato como fonte energética ${ }^{5}$.

Neste sentido, os videogames ativos (VGAs) surgem como opção de atividade física, visto que com sua prática é capaz de gerar aumento nos valores da frequência cardíaca e gasto energético ${ }^{12-14}$, além de causar mudanças na composição corporal ${ }^{15}$. No entanto, apesar da realidade virtual ser utilizada como instrumento para aumento da atividade física diária, ainda é insatisfatório o conhecimento dessa ferramenta como modificador dos níveis elevados da glicemia em idosos com DM.

Sendo assim, o objetivo do presente estudo foi de realizar uma revisão integrativa sobre o treinamento com videogames ativos em idosos com DM 2.

\section{MATERIAL E MÉTODOS}

Empregou-se a revisão integrativa, a qual buscou informações de pesquisas originais com metodologias semelhantes, com o objetivo de verificar a influência do treinamento com VGAs em uma população idosa com DM.

As buscas dos artigos científicos foram realizadas no ano de 2014, por dois pesquisadores de maneira independente, nas seguintes bases de dados eletrônicas: Bireme ${ }^{\circledR}$ (http://www.bireme.br), PubMed ${ }^{\circledR}$ (http://www.ncbi.nlm.nih.gov/pubmed), ScienceDirect ${ }^{\circledR}$ (http://www.sciencedirect.com) e Web of Knowledge $\mathbb{}$ (http://www.isiknowledge.com). Utilizando os termos e descritores: "exergames", "exercise games", "video game", "virtual reality", "diabetes mellitus" e "glycosyleted hemoglobin", mediante consulta ao MeSH - Medical Subject Headings e ao DeCS Descritores em Ciências da Saúde, por meio da Biblioteca Virtual em Saúde (BVS). Além disso, recorreu-se ao operador "AND" para combinação dos descritores e termos utilizados para busca das publicações.

Após o procedimento de busca, foram identificados inicialmente 415 manuscritos. Em seguida, os artigos que atenderam os critérios de inclusão: a) intervenção com videogames ativos; b) amostra com DM1 e/ou DM2; c) artigos originais de pesquisa com idosos acima de 60 anos, foram adicionados à pesquisa. Para tanto, foram excluídos: a) artigos de revisão, teses, dissertações, monografia e duplicatas.

\section{RESULTADOS}

Inicialmente foi realizada a leitura do título e resumo para identificação de artigos elegíveis. Após essa primeira análise, foram excluídos 411 manuscritos por não se adequarem aos critérios de inclusão da pesquisa. Em seguida, foram considerados elegíveis para a segunda fase desta revisão 4 estudos, os quais 2 
foram excluídos por serem duplicatas. Posteriormente, os artigos selecionados foram lidos na íntegra e como análise adicional, as referências dos artigos selecionados foram lidas com o objetivo de verificar potenciais artigos para seleção do presente estudo. Entretanto, nenhum artigo foi selecionado, resultando em um total de dois artigos que investigaram o efeito da intervenção com VGAs, publicados no ano de 2013. No quadro 1, é apresentado o processo de busca e seleção dos artigos nas bases de dados eletrônicas.

Entre os artigos incluídos, um foi realizado na Alemanha ${ }^{16}$ e o outro na Coréia do Sul ${ }^{17}$, utilizando jogos diferentes, o que torna difícil a comparação entre eles. Além disso, os estudos envolveram intervenções que duraram de 10 a 12 semanas, com uma população de idosos, apresentando um tamanho amostral entre 55 e 220 sujeitos dos dois gêneros, inativos fisicamente e diagnosticados com DM 2.

As características dos artigos incluídos nesta revisão, destacando o autor, amostra, objetivo, console dos videogames e jogo utilizado, tempo de intervenção e principais resultados estão apresentados a seguir na Tabela 1.

Observa-se que um estudo analisou as variáveis metabólicas e antropométricas ${ }^{16}$. Assim, os resultados encontrados indicam haver associação entre a prática de VGAs com a melhora dessas medidas em idosos. Possibilitando aumento no nível de atividade física dos participantes após uma intervenção de trinta minutos diários durante doze semanas.

\begin{tabular}{|c|c|c|c|}
\hline \multicolumn{4}{|c|}{ Quadro 1. Resultados das estratégias de busca dos artigos nas bases de dados eletrônicas (2014) } \\
\hline $\begin{array}{c}\text { Base de } \\
\text { dados }\end{array}$ & Palavras-Chave & $\begin{array}{c}\text { Resultado } \\
\text { da busca }\end{array}$ & $\begin{array}{c}\text { Artigos } \\
\text { Selecionados }\end{array}$ \\
\hline \multirow[t]{8}{*}{ Bireme } & "exergames" AND “diabetes mellitus" & 2 & 0 \\
\hline & "exergames" AND "glycosyleted hemoglobin" & 0 & 0 \\
\hline & "exercise games" AND "diabetes mellitus" & 0 & 0 \\
\hline & "exercise games" AND "glycosyleted hemoglobin" & 0 & 0 \\
\hline & "video games" AND "diabetes mellitus" & 4 & 0 \\
\hline & "video games" AND "glycosyleted hemoglobin" & 0 & 0 \\
\hline & "virtual reality AND diabetes mellitus" & 14 & 0 \\
\hline & "virtual reality AND alycosvlated hemoglobin" & 0 & 0 \\
\hline \multirow[t]{8}{*}{ Pub Med } & "exergames" AND "diabetes mellitus" & 2 & 0 \\
\hline & "exergames" AND "glycosyleted hemoglobin" & 0 & 0 \\
\hline & "exercise games" AND "diabetes mellitus" & 11 & 1 \\
\hline & "exercise games" AND "glycosyleted hemoglobin" & 3 & 0 \\
\hline & "video games" AND "diabetes mellitus" & 3 & 0 \\
\hline & "video games" AND "glycosyleted hemoglobin" & 1 & 0 \\
\hline & "virtual reality AND diabetes mellitus" & 15 & 1 \\
\hline & "virtual reality AND qlycosvlated hemoqlobin" & 3 & 0 \\
\hline \multirow{8}{*}{$\begin{array}{l}\text { Web of } \\
\text { Knowledge }\end{array}$} & "exergames" AND "diabetes mellitus" & 0 & 0 \\
\hline & "exergames" AND "glycosyleted hemoglobin" & 0 & 0 \\
\hline & "exercise games" AND “diabetes mellitus" & 1 & 1 \\
\hline & "exercise games" AND "glycosyleted hemoglobin" & 0 & 0 \\
\hline & "video games" AND “diabetes mellitus" & 1 & 0 \\
\hline & "video games" AND "glycosyleted hemoglobin" & 1 & 0 \\
\hline & "virtual reality AND diabetes mellitus" & 2 & 1 \\
\hline & "virtual realitv AND alycosvlated hemoglobin" & 0 & 0 \\
\hline \multirow[t]{8}{*}{ Science Direct } & "exergames" AND “diabetes mellitus" & 5 & 0 \\
\hline & "exergames" AND "glycosyleted hemoglobin" & 0 & 0 \\
\hline & "exercise games" AND "diabetes mellitus" & 5 & 0 \\
\hline & "exercise games" AND "glycosyleted hemoglobin" & 0 & 0 \\
\hline & "video games" AND "diabetes mellitus" & 60 & 0 \\
\hline & "video games" AND "glycosyleted hemoglobin" & 4 & 0 \\
\hline & "virtual reality AND diabetes mellitus" & 262 & 0 \\
\hline & "virtual reality AND glycosvlated hemoglobin" & 16 & 0 \\
\hline
\end{tabular}

Total: 415; Selecionados: 4; Duplicados: 2; Incluídos: 2 


\begin{tabular}{|c|c|c|c|c|c|}
\hline $\begin{array}{l}\text { Autor } \\
\text { (ano) }\end{array}$ & $\begin{array}{c}\text { Amostra } \\
\text { (idade) }\end{array}$ & Objetivo & Console (jogo) & $\begin{array}{l}\text { Tempo de } \\
\text { intervencão }\end{array}$ & Principais Resultados \\
\hline $\begin{array}{l}\text { (Kempf } \\
\text { and } \\
\text { Martin, } \\
\text { 2013). }\end{array}$ & $\begin{array}{c}\mathrm{N}=220 \\
50-75 \\
\text { anos }\end{array}$ & $\begin{array}{l}\text { Analisar os efeitos do } \\
\text { videogame ativo sobre o } \\
\text { controle metabólico e } \\
\text { qualidade de vida em } \\
\text { diabéticos do Tipo } 2\end{array}$ & $\begin{array}{l}\text { Nintendo Wii } \\
\text { (Wii Fit Plus) }\end{array}$ & 12 semanas & $\begin{array}{l}\text { Reduções significativas } \\
\text { na hemoglobina glicada } \\
\text { (Hb1A1c) após } 12 \\
\text { semanas de intervenção } \\
\text { com VGA. } \\
\text { Redução do Índice de } \\
\text { Massa Corporal (IMC) e } \\
\text { aumento do nível de } \\
\text { atividade física } \\
\text { siqnificativas no GE }\end{array}$ \\
\hline $\begin{array}{l}\text { (Lee } \\
\text { and } \\
\text { Shin, } \\
\text { 2013). }\end{array}$ & $\begin{array}{c}N=55 \\
X \text { idade= } \\
65 \text { anos }\end{array}$ & $\begin{array}{l}\text { Analisar o efeito de } 10 \\
\text { semanas de intervenção } \\
\text { com VGA sobre o } \\
\text { equilibrio, força, marcha e } \\
\text { eficácia contra quedas em } \\
\text { idosos diabéticos do Tipo } \\
2 .\end{array}$ & $\begin{array}{c}\text { PlayStation } 2 \\
\text { (“1.Wishi Washi": } \\
\text { Window Washing," } \\
\text { "2.Keep Ups: } \\
\text { Heading Game," } \\
\text { "3.Bowling," } \\
\text { "4.Bubble Pop," } \\
\text { "5.Boot Camp," "6. } \\
\text { Kuna Foo"). }\end{array}$ & 10 semanas & $\begin{array}{l}\text { Foram verificadas } \\
\text { melhoras significativas no } \\
\text { equilíbrio, força, marcha e } \\
\text { eficácia contra quedas na } \\
\text { população estudada após } \\
\text { a intervenção com VGA's. }\end{array}$ \\
\hline
\end{tabular}

N: amostra: X idade: média de idade: GE: Grupo experimental; GC: Grupo controle

\section{DISCUSSÃO}

Alternativas tecnológicas, já são utilizadas desde a década de 90 no tratamento da DM, através de jogos educacionais com o objetivo de orientar os pacientes diabéticos a manter hábitos de vida saudáveis, controlando aspectos nutricionais e condutas esportivas, visando o controle glicêmico ${ }^{18}$. Dessa forma, observa-se que ao analisar o efeito de seis semanas de intervenção com o jogo educativo "Packy \& Marlon" em pacientes com DM 1, esse tempo de prática foi eficiente em provocar diminuições nos níveis glicêmicos desses pacientes, além de causar um decréscimo na frequência de internação clínica ${ }^{19}$. Assim, devido ao envolvimento e participação nesses jogos, os praticantes são capazes de se concentrar mais durante a sessão, e estimular mais o sistema nervoso simpático, possibilitando maior captação da glicose após uma partida de videogame sedentário em sujeitos com DM $1^{20}$.

Por outro lado, ao analisar pacientes diagnosticados com DM 2, uma pesquisa realizada no Brasil $^{10}$ indicou que 20 semanas de intervenção com três ou cinco sessões de treinamento aeróbio semanal não são eficientes em diminuir os valores da $\mathrm{HbA1c}$ desses sujeitos. No entanto, de maneira contrária, verifica-se que com apenas 12 semanas de intervenção com videogames ativos, é capaz de promover diminuição significativa nos níveis de glicemia em jejum e da $\mathrm{HbAlc}$ em idosos com DM 2. Demonstrando que um programa com videogame ativo é capaz de produzir efeitos positivos em relação a uma intervenção estruturada ${ }^{16}$.

Deve-se levar em consideração que os resultados obtidos por pacientes com DM, nem sempre serão alcançados por pessoas não diabéticas. Isso foi verificado agudamente após uma sessão de treinamento com VGAs, no qual, os sujeitos analisados não apresentaram mudanças significativas na variável glicemia. Possivelmente o fato da amostra ser normoglicêmica ocorreu a gliconeogênese, impedindo que os valores da glicemia diminuíssem após a sessão $0^{3,21}$.

De acordo com os resultados obtidos na busca da literatura, constatou-se que poucos estudos utilizam os VGAs como alternativa coadjuvante no tratamento da DM. Porém, observou-se nesta revisão, que a 
intervenção com VGAs com uma frequência mínima de duas vezes por semana, durante um período de dez semanas, é suficiente para reduzir os valores da hemoglobina glicada e os riscos de adquirir outras doenças durante o processo de envelhecimento. Além de proporcionar melhoras significativas no equilíbrio ${ }^{17}$, o qual apresenta associação na evolução de força e potência dos membros inferiores ${ }^{2}$.

A principal limitação do presente estudo foi a não utilização de jogos específicos para população de idosos com DM.

\section{CONCLUSÃO}

Os VGAs apresentam características positivas no tratamento não farmacológico da DM em idosos, pois promovem auto regulação na taxa de glicose e diminuição da hemoglobina glicada dos sujeitos com DM 2. Além disso, reduzem o declínio funcional, gerando melhoras no equilíbrio. No entanto, são necessários estudos futuros que possam identificar os efeitos dos VGAs a longo prazo e com um maior número de sujeitos, a partir de sessões experimentais randomizadas e controladas.

\section{REFERÊNCIAS}

1. Matsudo SM, Keihan V, Matsudo R. Impacto do envelhecimento nas variáveis antropométricas, neuromotoras e metabólicas da aptidão física. Rev Bras Ciência e Mov. 2000; 8(4):21-32.

2. Chodzko-Zajko WJ, Proctor DN, Fiatarone Singh $M$ a, Minson CT, Nigg CR, Salem GJ, et al. American College of Sports Medicine position stand. Exercise and physical activity for older adults. Med Sci Sports Exerc. 2009; 41(7):1510-1530.

3. Douglas CR. Fisiologia Aplicada à Nutrição, 2 ed. - Rio de Janeiro: Guanabara Koogan. Journal of the Royal Society of Medicine. 2006.

4. Alvarenga K de F, Duarte JL, Silva DPC da, AgostinhoPesse RS, Negrato CA, Costa OA. Potencial cognitivo P300 em indivíduos com diabetes mellitus. Rev Bras Otorrinolaringol. 2005; 71(2):202-207.

5. American Diabetes Association A. Standard of medical care in diabetes-2011. Diabetes Care. 2011; 34(1):S1161.

6. SBD D. Diretrizes da Sociedade Brasileira de Diabetes. Rio de Janeiro: Diagraphic. 2006.

7. Passos VM de A, Barreto SM, Diniz LM, Lima-Costa MF. Type 2 diabetes/: prevalence and associated factors in a Brazilian community - the Bambuí health and aging study. 2005;123(2):66-71.

8. Vancea DMM, Vancea JN, Pires MIF, Reis MA, Moura RB, Dib SA. Artigo Original Efeito da Freqüência do Exercício Físico no Controle Glicêmico e Composição Corporal de Diabéticos Tipo 2. Soc Bras Cardiol. 2009; 92(1):23-30.

9. Dunstan DW, Daly RM, Owen N, Jolley D, De Courten M, Shaw J, et al. High-intensity resistance training improves glycemic control in older patients with type 2 diabetes. Diabetes Care. 2002; 25(10):1729-1736.

10. Kraemer WJ, Steven. Fisiologia do Exercício. Teoria \& Prática. Guanabara Koogan. 2013.

11. McArdle WD, Katch FI, Kantch VL. Fisiologia do exercício: energia, nutrição e desempenho humano. 5ed. Rio de Janeiro: Guanabara Koogan. 2003.
12. Graves LEF, Ridgers ND, Williams K, Stratton G, Atkinson G, Cable NT. The physiological cost and enjoyment of Wii Fit in adolescents, young adults, and older adults. J Phys Act Health. 2010; 7(3):393-401.

13. Smallwood SR, Morris MM, Fallows SJ, Buckley JP. Physiologic responses and energy expenditure of kinect active video game play in schoolchildren. Arch Pediatr Adolesc Med. 2012;166(11):1005-1009.

14. Brito-gomes JL, Perrier-melo RJ, Albuquerque FL de, Costa $\mathrm{M}$ da C. Comportamento da frequência cardíaca durante uma sessão com diferentes vídeo games ativos. Man Ther posturology Reabil J. 2014; 11(55)81-95.

15. Adamo KB, Rutherford J a, Goldfield GS. Effects of interactive video game cycling on overweight and obese adolescent health. Appl Physiol Nutr Metab. 2010; 35(6):805-815.

16. Kempf K, Martin S. Autonomous exercise game use improves metabolic control and quality of life in type 2 diabetes patients - a randomized controlled trial. BMC Endocr Disord. 2013;13(57):1-9.

17. Lee $\mathrm{S}$, Shin S. Effectiveness of virtual reality using video gaming technology in elderly adults with diabetes mellitus. Diabetes Technol Ther. 2013;15(6):489-496.

18. Lieberman $D$ a. Video games for diabetes selfmanagement: examples and design strategies. J Diabetes Sci Technol. 2012; 6(4):802-806.

19. Brown SJ, Lieberman DA, Gemeny BA, Fan YC, Wilson DM, Pasta DJ. Educational video game for juvenile diabetes: Results of a controlled trial. Med Informatics. 1997; 22 (1):77-89.

20. Phan-Hug F, Thurneysen E, Theintz G, Ruffieux C, Grouzmann E. Impact of videogame playing on glucose metabolism in children with type 1 diabetes. Pediatr Diabetes. 2011;12(8):713-717.

21. Soltani P, Salesi M. Effects of Exergame and Music on Acute Exercise Responses to Graded Treadmill Running. Games Health J. 2013; 2(2):75-80. 
22. Liese AD, Ma X, Maahs DM, Trilk JL. Physical activity, sedentary behaviors, physical fitness, and their relation to health outcomes in youth with type 1 and type 2 diabetes: A review of the epidemiologic literature. J Sport Heal Sci.; 2013; 2(1):21-38.
Correspondência

Raphael José Perrier Melo

R. Arnóbio Marques, 310, Santo Amaro,

Campus HUOC - CEP: 50.100-130

Recife - Pernambuco - Brasil

E-mail: rperrier2@gmail.com

raphael_perrier90@hotmail.com 